\title{
LES VARIATIONS RAPIDES DU CLIMAT DANS L'OCÉAN ATLANTIQUE NORD AU COURS DES 60000 DERNIÈRES ANNÉES
}

\author{
Elsa Cortijo \\ Laboratoire des sciences du climat et de l'environnement \\ (anciennement Centre des faibles radioactivités) \\ Domaine du CNRS \\ Avenue de la Terrasse \\ 91198 Gif-sur-Yvette Cedex
}

RÉSUMÉ

Les variations lentes du climat de notre planète, à l'échelle de quelques dizaines de milliers d'années, sont essentiellement dues aux changements réguliers des paramètres de l'orbite de la Terre autour du Soleil. Or, des études récentes effectuées sur des carottes de glace ont montré que certains changements climatiques pouvaient se produire de manière brutale, en quelques centaines voire en quelques dizaines d'années seulement.

Les variations climatiques rapides enregistrées dans les glaces au cours des 60000 dernières années apparaissent aussi dans les sédiments marins. L'étude détaillée des carottes de sédiments marins a ainsi permis de montrer que des épisodes de débâcles massives d'icebergs ont eu lieu au cours de la dernière période glaciaire, entre - 60000 et - 10000 ans, entraînant une baisse de la température et de la salinité de l'eau dans l'Atlantique nord, ainsi qu'un ralentissement de la circulation océanique. Ces variations de température - de 1 à $3{ }^{\circ} \mathrm{C}$ dans l'océan Atlantique nord en surface et de 5 à $8^{\circ} \mathrm{C}$ dans les masses d'air au-dessus du Groenland - représentent des changements climatiques considérables dans l'hémisphère nord.

ABSTRACT Rapid changes in the climate of the North Atlantic Ocean during the last 60000 years

The slow variations of the terrestrial climate, on time scales of some tens of thousands of years, are essentially due to regular changes in the Earth's orbital parameters. Recently, some studies on ice cores have shown that climatic changes can occur abruptly, on time scales of centuries or even decades.

The rapid climatic variations found in the ice are also recorded in the marine sediment. The detailed study of marine sediment shows that massive discharges of icebergs occurred several times during the last glacial period between 60000 and 10000 years ago. As a result, the North Atlantic underwent huge hydrological changes during this period, with decreases in temperature and salinity of its surface waters. The 1 to $3{ }^{\circ} \mathrm{C}$ temperature variations in the North Atlantic Ocean and 5 to $8^{\circ} \mathrm{C}$ temperature variations in the Greenland atmosphere represent huge climatic changes over the Northern hemisphere. 


\section{LES VARIATIONS CLIMATIQUES}

Figure 1 - Étendue des glaces continentales reconstituée par le groupe de travail Climap, 1981.

(a) au dernier maximum glaciaire (il y a environ 18000 ans) : (b) de nos jours.
Au cours du Quaternaire, c'est-à-dire depuis environ deux millions d'années, le climat de la Terre s'est caractérisé par une succession de longues périodes glaciaires, interrompues par de courtes périodes chaudes (dites interglaciaires) semblables à celle que nous connaissons actuellement. Le climat de la Terre a donc oscillé entre des périodes froides, pendant lesquelles les glaciers continentaux étaient très développés, et des périodes chaudes, pendant lesquelles ils n'avaient qu'une extension limitée (figure 1). C'est la découverte par Louis Agassiz au milieu du XIX ${ }^{\text {e }}$ siècle des traces laissées par ces glaciers continentaux qui a conduit l'astronome serbe Milutin Milankovitch à formuler, au début du XX siècle, la théorie astronomique des paléoclimats (encadré ci-après). Selon celleci, les variations du climat à des échelles de temps de l'ordre de plusieurs dizaines ou centaines de milliers d'années seraient gouvernées par les variations lentes des paramètres orbitaux de la Terre.
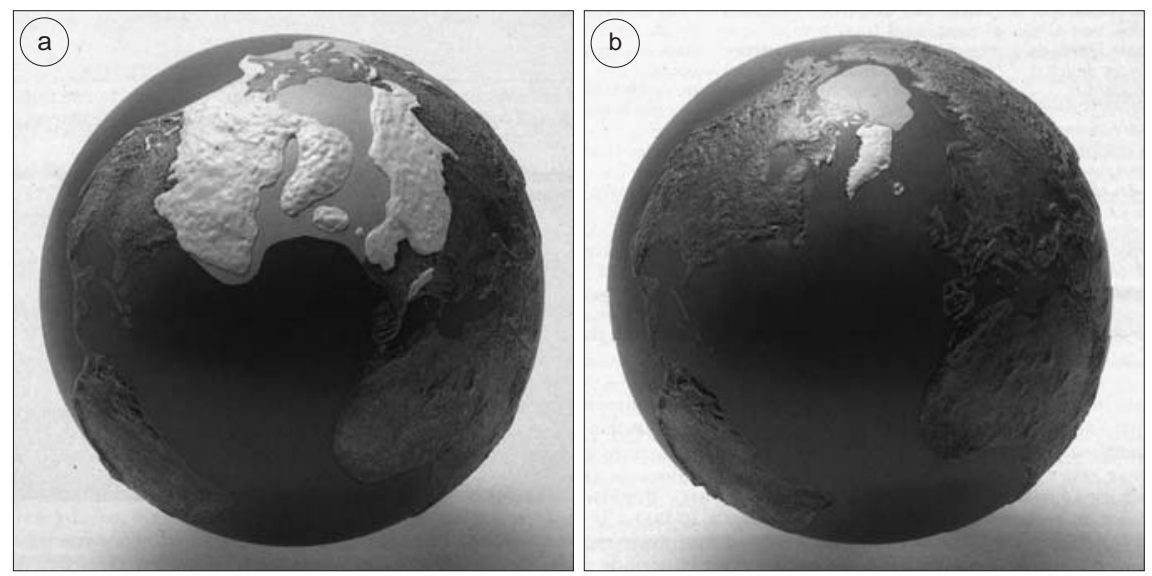

\section{La théorie astronomique des paléoclimats}

Les paramètres astronomiques suivants contrôlent les variations climatiques lentes, et donc les alternances période glaciaire période interglaciaire (figure 2):

- L'excentricité de l'écliptique représente le degré d'allongement de l'ellipse de l'orbite terrestre autour du Soleil. Les variations de ce paramètre jouent sur la quantité d'énergie reçue par la Terre avec une périodicité de l'ordre de 400000 ans. Une période d'environ 100000 ans y est également associée. Lorsque l'ellipse est plus allongée, la distance moyenne séparant la Terre du Soleil diminue et notre planète reçoit un petit peu plus d'énergie. Cependant, l'effet de ces modifications de l'excentricité est très faible en termes d'énergie et ne conduit au maximum qu'à une variation de température moyenne de quelques dixièmes de degrés.

- L'obliquité représente l'inclinaison de l'axe de la Terre sur le plan de son orbite. Ses variations (de $\pm 1^{\circ} 30^{\prime}$ autour d'une valeur moyenne de $23^{\circ} 30^{\prime}$ avec une périodicité de 41000 ans) ont une action sur le contraste thermique entre été et hiver. En effet, lorsque l'inclinaison est forte, les pôles pointent davantage vers le Soleil en été et les hautes latitudes reçoivent alors beaucoup plus de chaleur que lorsque I'inclinaison est faible. Cette configuration conduit donc à des étés chauds et des hivers froids aux hautes latitudes ; elle correspond ainsi plutôt aux climats interglaciaires, avec peu de glace aux hautes latitudes des continents.

- La précession des équinoxes représente la lente dérive de la position des équinoxes par rapport au périhélie et a pour effet de modifier la répartition saisonnière de l'insolation en

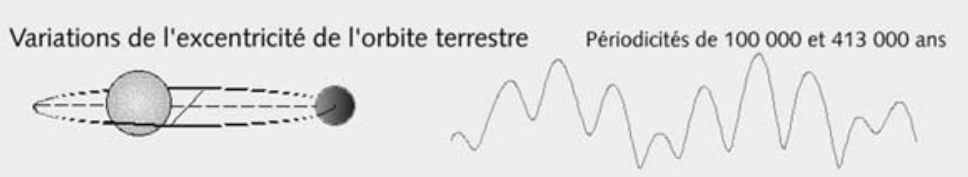

Variations de l'obliquité de l'axe de rotation

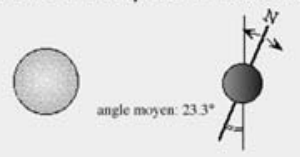

Périodicité de 41000 ans

Précession de l'axe de rotation et rotation de l'orbite terrestre

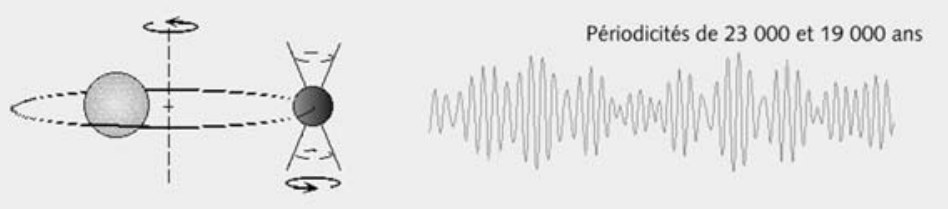

Figure 2 - Illustration des trois paramètres orbitaux de la Terre et des effets de leur variation sur le climat.

jouant sur la distance Terre-Soleil. Les périodes principales associées à ce phénomène sont de 19000 et 23000 ans. Aujourd'hui, la Terre est près du Soleil en décembre et loin en juillet. II y a 11000 ans, la Terre était loin du Soleil en décembre et connaissait donc des hivers plus froids dans I'hémisphère nord, mais près du Soleil en juillet d'où des étés plus chauds. 
Cette théorie a été confirmée par Hays et al. (1976), grâce à l'étude de marqueurs paléoclimatiques dans les sédiments marins. En effet, les différentes périodicités astronomiques se retrouvent dans la plupart des enregistrements paléoclimatiques (figure 4), ce qui suggère fortement que les variations climatiques à long terme sont gouvernées par les changements de l'orbite terrestre. Ces variations climatiques, à l'échelle des derniers milliers d'années, sont maintenant bien connues grâce à la mesure des variations de la composition isotopique de l'oxygène des coquilles de foraminifères trouvées dans les sédiments marins (encadré ci-après).

\section{Foraminifères, isotopes de l'oxygène et climat}

Les foraminifères planctoniques(1) sont des organismes unicellulaires évoluant dans les eaux superficielles de l'océan. Leurs coquilles carbonatées, qui s'accumulent dans les sédiments marins, sont un véritable thermomètre de l'océan à travers les âges. En effet, lors de son développement, le foraminifère utilise le carbonate de calcium dissous dans l'eau pour bâtir sa coquille. Or, les ions carbonate dissous dans l'eau de mer sont d'autant plus riches en ${ }^{18} \mathrm{O}$ que l'eau de mer elle-même en contient davantage. De plus, les ions carbonate contenant un atome ${ }^{18} \mathrm{O}$ ne précipitent pas à la même vitesse que ceux qui
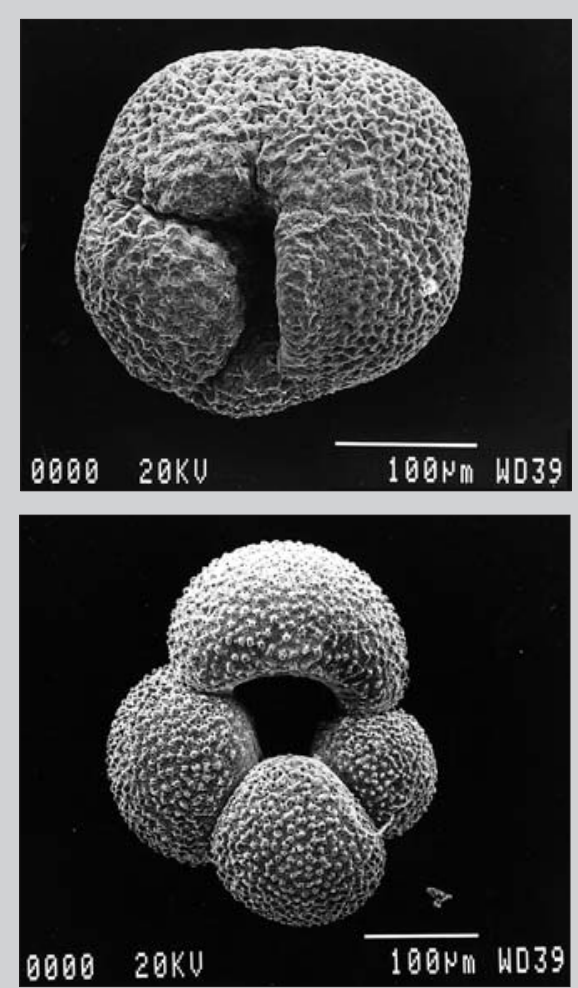

Deux espèces de foraminifères planctoniques. En haut, Neogloboquadrina pachyderma à enroulement senestre, espèce polaire supportant des eaux de 0 à $8{ }^{\circ} \mathrm{C}$.

En bas, Globigerina bulloides, espèce subpolaire supportant des eaux de 10 à $22^{\circ} \mathrm{C}$.

(C) LSCE) ne renferment que l'isotope léger ${ }^{16} \mathrm{O}$. Cette différence de vitesse est fonction de la température. En conséquence, le rapport ${ }^{18} \mathrm{O} /{ }^{16} \mathrm{O}$ (ou composition isotopique) des coquilles de foraminifères recueillies dans les sédiments marins dépend à la fois de la température et du rapport ${ }^{18} \mathrm{O} /{ }^{16} \mathrm{O}$ de l'eau dans laquelle ces animaux se sont développés. Ces différents paramètres sont reliés par une formule empirique (figure 3) connue sous le nom de « formule des paléotempératures » (Epstein et al., 1953).

Par ailleurs, les molécules d'eau contenant l'isotope léger ${ }^{16} \mathrm{O}$ s'évaporent plus vite que celles formées avec l'isotope lourd ${ }^{18} \mathrm{O}$. II en résulte que la vapeur d'eau atmosphérique est appauvrie en ${ }^{18} \mathrm{O}$ par rapport à l'eau de mer qui lui a donné naissance. Au contraire, les molécules d'eau comportant l'isotope ${ }^{18} \mathrm{O}$ ont davantage tendance à se condenser dans la pluie : aussi le rapport ${ }^{18} \mathrm{O} /{ }^{16} \mathrm{O}$ de la vapeur d'eau restant dans les nuages diminue-t-il en même temps que l'humidité de l'air. Les nuages qui arrivent dans les zones polaires sont ainsi déjà très appauvris en ${ }^{18} \mathrm{O}$ du fait des pluies qu'ils ont fournies sur leur trajet depuis les tropiques : les chutes de neige sur le Groenland sont appauvries en ${ }^{18} \mathrm{O}$ de $30 \%$ environ par rapport à l'eau de mer. Ainsi, lorsque le volume des glaces augmente, le rapport ${ }^{18} \mathrm{O} /{ }^{16} \mathrm{O}$ augmente dans l'eau des océans.

Les mesures du rapport ${ }^{18} \mathrm{O} /{ }^{16} \mathrm{O}$ des coquilles de foraminifères enregistrent donc simultanément les variations du rapport ${ }^{18} \mathrm{O} /{ }^{16} \mathrm{O}$ de l'eau de mer dues aux variations planétaires du volume de glace et les changements de température de l'eau. Les causes du signal climatique sont donc complexes, mais l'interprétation en est relativement simple puisque la croissance d'un glacier et un refroidissement de l'eau se traduisent tous deux par une augmentation du rapport ${ }^{18} \mathrm{O} /{ }^{16} \mathrm{O}$ des coquilles de foraminifères des sédiments marins ; inversement, lorsque la température de l'eau augmente et que les glaciers fondent, ce rapport ${ }^{18} \mathrm{O} /{ }^{16} \mathrm{O}$ diminue.

Les foraminifères planctoniques enregistrent tous les changements hydrologiques qui peuvent affecter les eaux de surface : changements planétaires du volume de glace, variations de température et de salinité de l'eau. Les foraminifères benthiques, quant à eux, n'enregistrent en grande partie que les changements planétaires, c'est-à-dire les variations mondiales du volume de glace.

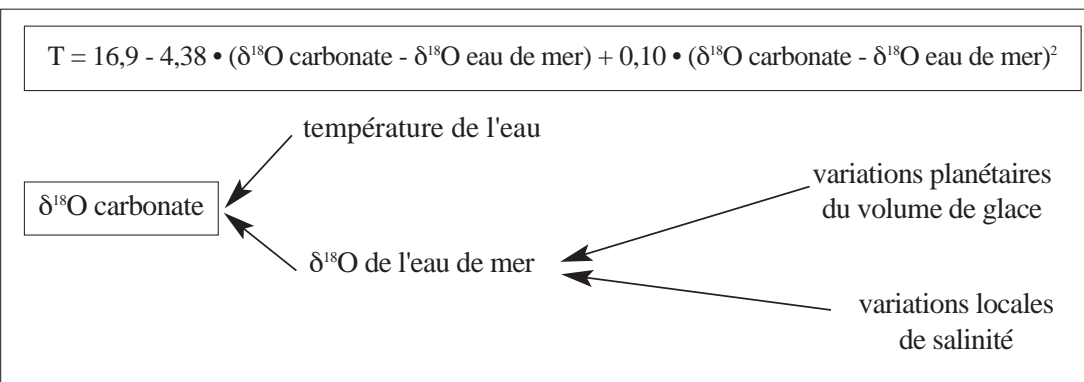

Figure 3 - Équation des paléotempératures formulée par Epstein et al. (1953) et modifiée par Shackleton (1974); schéma montrant les divers paramètres qui influencent la teneur en ${ }^{18} \mathrm{O}$ des coquilles de foraminifères planctoniques.

(1) Les expressions en caractères gras sont explicitées dans le glossaire à la fin de cet article. 


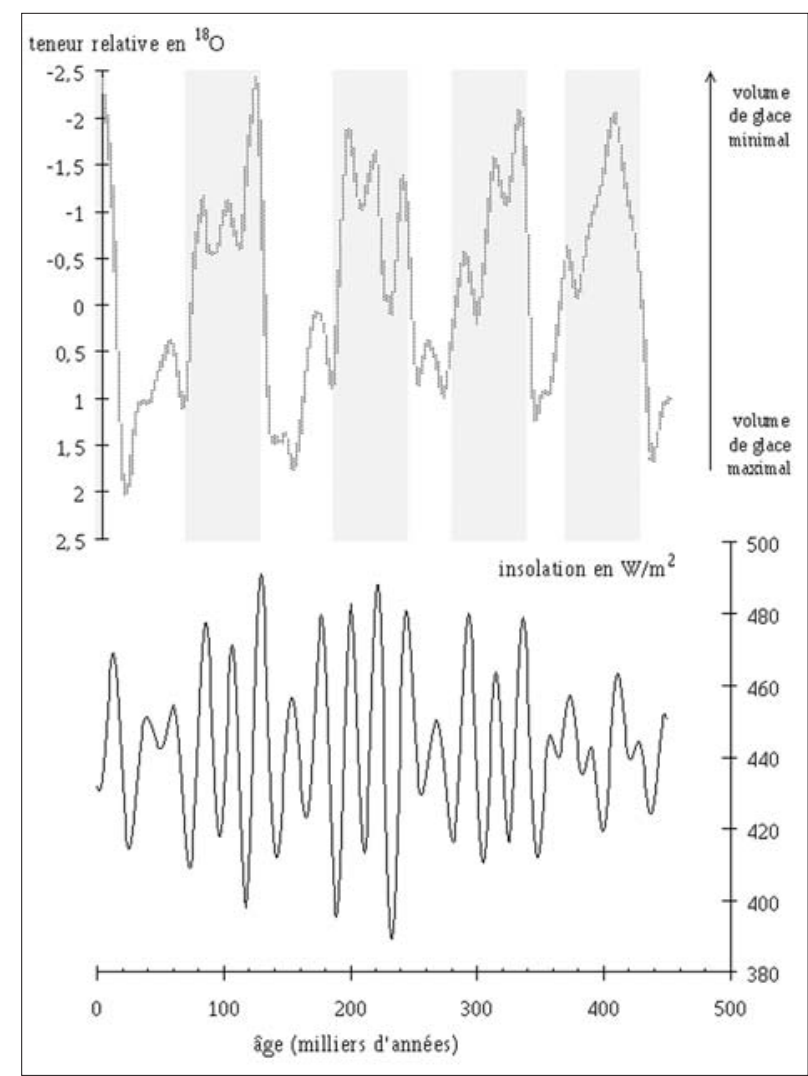

Figure 4 - Variations du volume de glace (exprimées en teneur relative en ${ }^{18} \mathrm{O}$ des coquilles de foraminifères des sédiments marins) sur plusieurs cycles climatiques, soit 450000 ans, (en haut) comparées aux variations de l'insolation calculées pour la même période (en bas). Les bandes grises soulignent les périodes interglaciaires, pendant lesquelles le volume de glace est minimal.
Il y a quelques années, les études paléoclimatiques étaient effectuées avec une résolution temporelle de l'ordre de quelques milliers d'années. En effet, on pensait que les phénomènes de bioturbation dans les sédiments profonds empêchaient la conservation de toute trace de changement rapide. Ces études ont néanmoins permis la définition des grands cycles glaciaire-interglaciaire d'un point de vue isotopique et sédimentaire (Emiliani, 1955). Les recherches conduites par Ruddiman en Atlantique nord (Ruddiman and McIntyre, 1981) ont montré que la quantité de matériel détritique relâchée par les icebergs dans le sédiment était étroitement liée à la taille des grandes calottes de glace de l'hémisphère nord. Bien que la résolution temporelle de l'échantillonnage n'excédât pas quelques milliers d'années, ce travail a permis d'établir des relations entre la nature de la sédimentation et les stades climatiques définis par la stratigraphie isotopique (voir l'encadré sur les méthodes de datation page 19).

Les carottes de glace sont exemptes de problèmes de bioturbation; aussi, les analyses faites dans les carottes de glace du Groenland ont rapidement révélé l'existence, entre - 60000 et - 10000 ans, d'une succession d'événements climatiques de large amplitude et d'une durée suffisamment courte (de l'ordre de quelques centaines d'années) pour qu'ils ne puissent être engendrés par les variations des paramètres orbitaux (Dansgaard et al., 1982). Ces oscillations, appelées événements de DansgaardOeschger et qui témoignent d'excursions atmosphériques plus chaudes au-dessus du Groenland, ont été confirmées dans les forages de glace plus récents (Dansgaard et al., 1993).

Depuis la fin des années quatre-vingt, les études paléoclimatiques ont bénéficié de l'augmentation de la résolution temporelle des analyses des sédiments marins. Cela a permis de montrer qu'il existait, au cours de la dernière période glaciaire (entre - 60000 et - 10000 ans), des variations brutales de la quantité de matériel détritique transportée par les icebergs, et cela indépendamment des fluctuations à long terme de la taille des calottes de glace (Broecker et al., 1992). Le climat de la Terre apparaît donc de plus en plus comme un système complexe caractérisé par des variations rapides, sans doute dues à des oscillations internes du système climatique, puisque les échelles de temps impliquées dans la théorie astronomique ne peuvent en rendre compte directement. Des mécanismes plus complexes, faisant probablement intervenir des interactions entre atmosphère, biosphère, océan et calottes de glace, doivent alors être invoqués.

Figure 5 - Carte de localisation des différents sites étudiés dans l'Atlantique nord. La carotte de glace Grip prélevée au Groenland figure aussi. Les sites marqués par des étoiles ont été étudiés au cours de ce travail, alors que ceux marqués par des points ont été étudiés par d'autres scientifiques: Bond et al. (1992 et 1993), Fillon et Duplessy (1980), Fronval et al. (1995), Hillaire-Marcel et al. (1994), Maslin et al. (1995), Mix et Fairbanks (1985), Rasmussen et al. (1996b), Van Kreveld et al. (1996). 


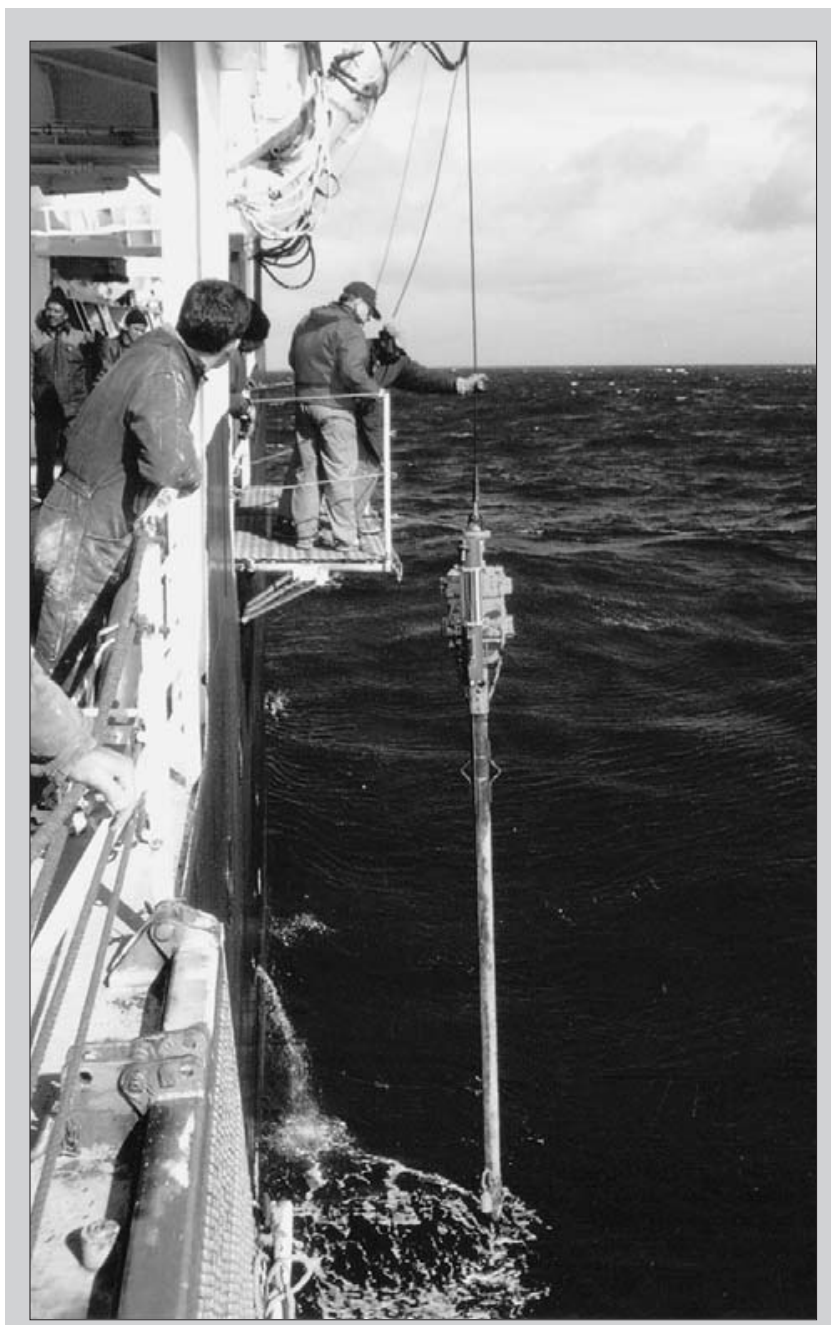

Opération de carottage en mer. (๑) LSCE)

\section{Carottes de sédiments marins, carottes de glace}

Les reconstitutions paléoclimatiques peuvent être obtenues, soit à partir de sédiments marins ou lacustres, soit à partir de glaces polaires. Les sédiments marins sont prélevés par la technique du carottage. Les navires océanographiques sont équipés d'un système constitué d'un tube de 10 à $60 \mathrm{~m}$ de

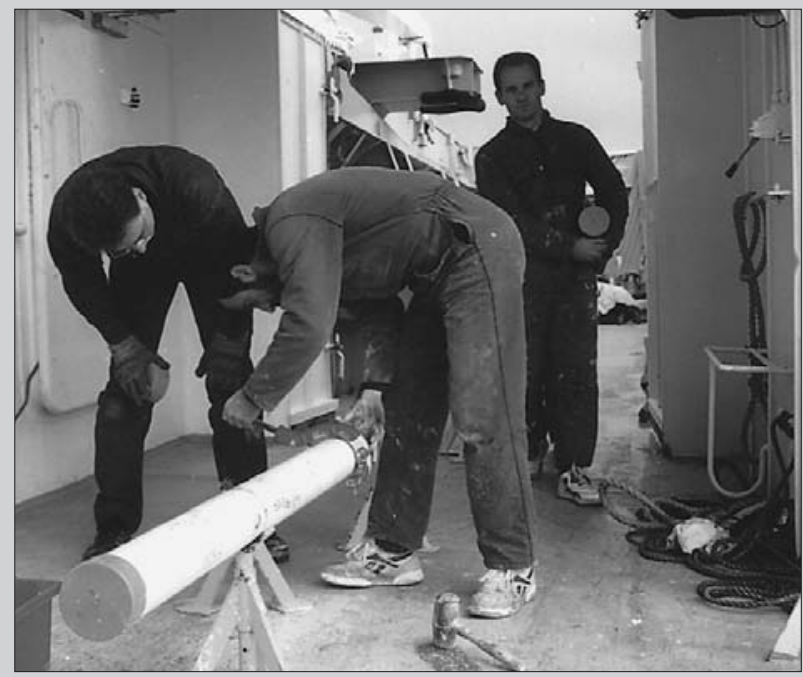

Manipulation des carottes sédimentaires sur le pont d'un navire océanographique. (C) LSCE)

long surmonté d'une masse de plusieurs centaines de kilogrammes. Les sites de carottage sont choisis en fonction d'informations déjà connues (carottages préalables, position par rapport aux courants marins, etc.) et des résultats du suivi en continu de l'épaisseur des sédiments marins par les méthodes sismiques au cours de la campagne océanographique. Lorsque la position du site est choisie, le carottier est mis à l'eau et, lorsqu'il atteint le sédiment, il s'y enfonce par gravité avant d'être remonté à bord. Pendant la remontée à bord, un système de clapets à la base de la carotte assure le maintien du sédiment dans le carottier. Une fois sur le pont du navire, la carotte est découpée en sections de longueur $150 \mathrm{~cm}$; puis chaque section est ouverte en deux demi-cylindres, l'un servant à faire les prélèvements aux fins d'analyses, l'autre étant conservé en archives.

L'étude des sédiments marins permet de reconstituer les variations du volume des glaces continentales, les variations de température et de salinité dans l'océan, les changements de la circulation océanique, mais aussi les variations de production biologique. Les études à partir des glaces continentales permettent, quant à elles, de reconstituer les variations de la température de l'air, celles des concentrations de différents gaz ainsi que d'autres paramètres atmosphériques. L'étude de ces deux types de matériau est donc parfaitement complémentaire.

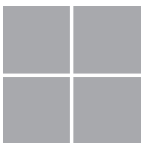

Deux types de variabilité rapide vont être détaillés dans la suite de cet article : - Les événements dont la périodicité est de l'ordre de 7000 à 10000 ans, enregistrés dans les sédiments de l'Atlantique nord entre 40 et 65 degrés nord (événements de Heinrich).

- Les événements dont la périodicité est de l'ordre de 1500 à 2000 ans, plus spécifiquement enregistrés dans les sédiments de la mer de Norvège (événements de Dansgaard-Oeschger).

Les carottes de sédiment marin (figure 5) ayant servi de base à cette étude proviennent de diverses missions océanographiques, essentiellement les missions Paleocinat 1 et 2 effectuées sur le navire océanographique de l'Ifremer, Le Suroît. 
CADRE STRATIGRAPHIQUE DE L'ÉTUDE

igure 6 - Construction d'une échelle d'âge. Exemple de la carotte SU90-08 $\left(43^{\circ} \mathrm{N}, 30^{\circ} \mathrm{W}\right)$

En haut, relation âge-profondeur obtenue à partir des différents marqueurs stratigraphiques

(carbone 14 : petites barres horizontales : niveau de cendres 2 : croix : transition entre stades isotopiques : étoile)

En bas, application de l'échelle d'âge à la composition isotopique de l'oxygène des foraminifères (attention, l'échelle des ordonnées va dans le sens des teneurs décroissantes).
L'étude d'événements climatiques rapides, dont la durée n'excède pas 2000 ans, à travers tout l'Atlantique nord implique la construction d'un cadre stratigraphique précis pour permettre des comparaisons spatiales et temporelles entre les différents enregistrements. Il est en effet indispensable de déterminer le mieux possible les échelles d'âge des différentes carottes de sédiments, de façon à pouvoir discuter des éventuels déphasages entre les diverses zones géographiques. L'établissement d'une chronologie précise est donc nécessaire pour cette étude.

Les méthodes de datation (encadré page 19) utilisées pour les sédiments marins sont, par ordre de priorité :

- les datations au radiocarbone disponibles pour les 40000 dernières années ;

- la stratigraphie isotopique de l'oxygène des foraminifères benthiques et les âges adoptés par le groupe Specmap (Martinson et al., 1987) pour les périodes antérieures à - 60000 ans ;

- les niveaux de cendres volcaniques datés et reconnus dans l'Atlantique nord.

À partir de ces différentes datations, nous avons construit, pour chaque carotte étudiée, une relation faisant correspondre profondeur et âge. Cette relation constitue une échelle d'âge qui a été appliquée aux différents paramètres enregistrés dans chaque carotte (figure 6). Cela nous a permis de montrer que les événements climatiques rapides pouvaient avoir des durées de l'ordre de quelques centaines d'années (à comparer aux échelles de temps des alternances glaciaireinterglaciaire : les dizaines de milliers d'années). On sait par ailleurs, d'après les études menées dans les glaces du Groenland, que de tels changements se produisent en fait en quelques dizaines d'années seulement. Une telle résolution temporelle est encore difficile à obtenir dans les sédiments marins.

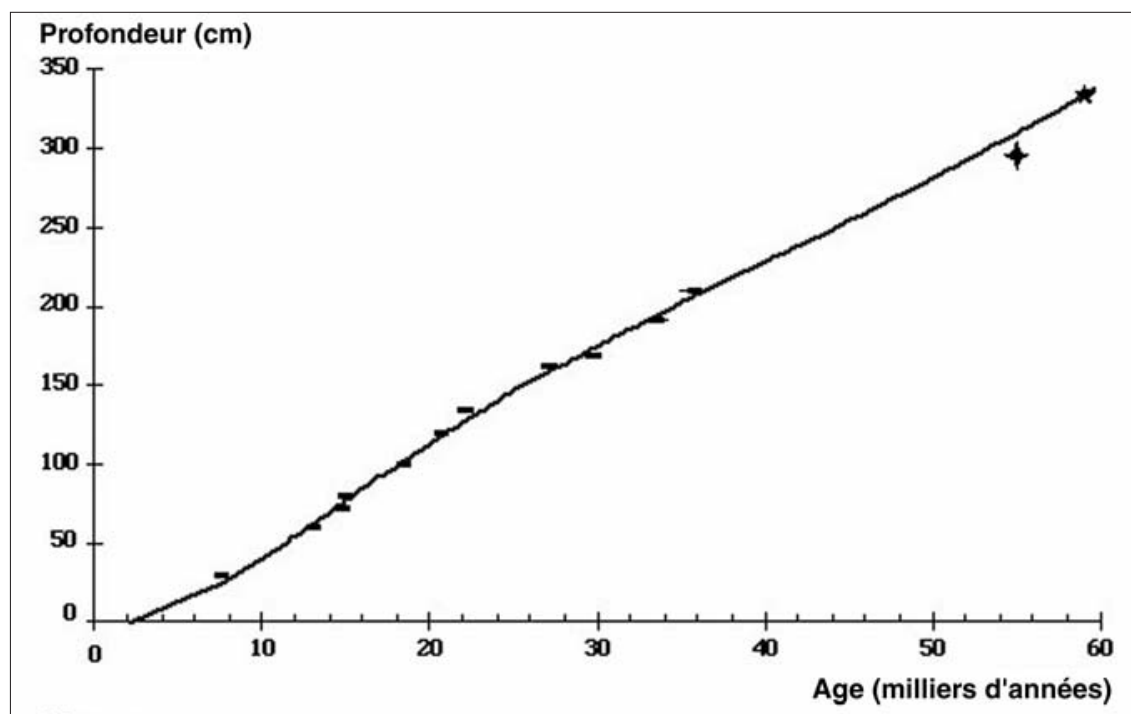

$\delta 180(\% 6)$

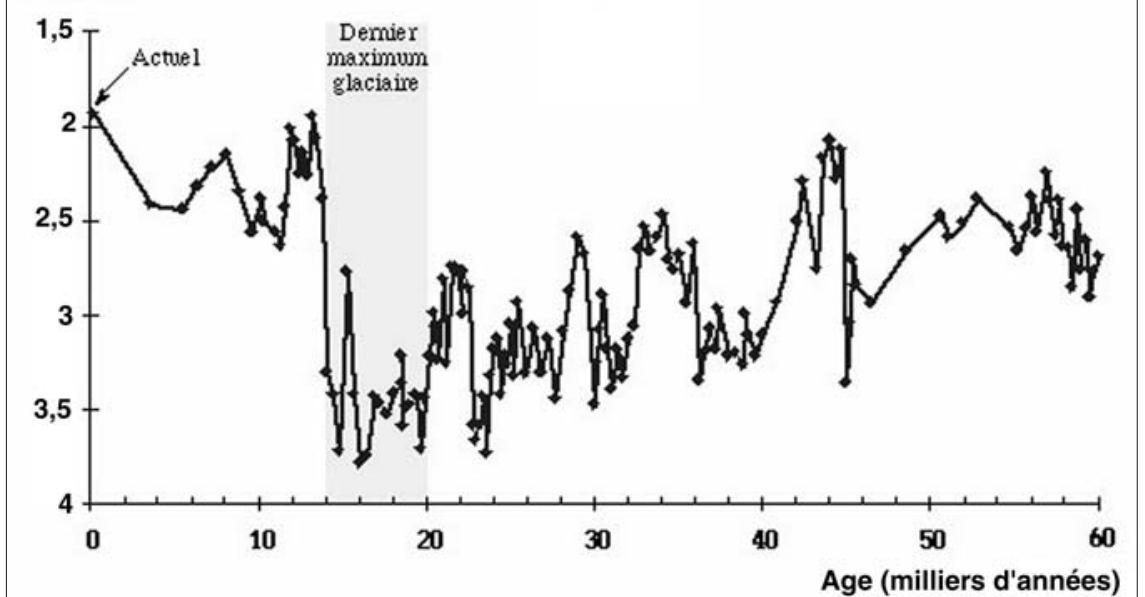




\section{Les méthodes de datation des sédiments marins}

- Les datations par le carbone 14 sont obtenues sur un milligramme de carbone par la mesure du rapport ${ }^{14} \mathrm{C} /{ }^{12} \mathrm{C}$ d'échantillons de foraminifères planctoniques d'une espèce donnée, en spectrométrie de masse couplée à un accélérateur situé à Gif-sur-Yvette. Cette méthode est utilisable pour les 40000 dernières années, avec une précision allant de la dizaine d'années pour les âges les plus jeunes à quelques centaines d'années autour de - 40000 ans. La mise en œuvre est assez longue, puisqu'il faut réunir environ $5 \mathrm{mg}$ de foraminifères d'une espèce (soit 1500 à 2000 individus d'environ $200 \mu \mathrm{m}$ ) pour obtenir une mesure.

- La stratigraphie isotopique repose sur la mesure du rapport ${ }^{18} \mathrm{O} /{ }^{16} \mathrm{O}$ des coquilles de foraminifères benthiques, c'est-à-dire vivant sur le fond des océans. Dans ce cas, ce rapport enregistre essentiellement les variations planétaires du volume des glaces, dont dépend le rapport ${ }^{18} \mathrm{O} /{ }^{16} \mathrm{O}$ moyen de l'océan, et qui sont contrôlées par les variations des paramètres astronomiques de la Terre. Les variations de l'insolation peuvent être calculées en fonction du temps et permettre ainsi d'ajuster une échelle d'âge sur les variations climatiques planétaires telles qu'elles sont reconstruites par le rapport ${ }^{18} \mathrm{O} /{ }^{16} \mathrm{O}$ des foraminifères benthiques (figure 4). C'est le travail auquel s'est livré le groupe Specmap (Martinson et al., 1987) ; le résultat est une courbe isotopique synthétique datée pour les derniers 700000 ans.

- Deux niveaux de cendres volcaniques sont enregistrés et datés dans les sédiments de I'Atlantique nord. Ils proviennent de deux grandes explosions volcaniques identifiées sur les continents : le niveau de cendres 1 est daté au carbone 14 à - 11000 ans environ, à partir de l'analyse de dépôts d'origine continentale et de foraminifères entourant le dépôt de cendres (Bard et al., 1994); le niveau de cendres 2 est daté par stratigraphie isotopique à - 55000 ans environ (Ruddiman and Glover, 1972). Ces niveaux, une fois repérés dans les sédiments marins, sont donc utilisables comme marqueurs stratigraphiques.

\section{LES ÉVÉNEMENTS DE HEINRICH}

Divers auteurs (Pastouret et al., 1975 ; Heinrich, 1988) ont mis en évidence, au sud-ouest du Groenland et au centre de l'Atlantique nord, des niveaux sédimentaires riches en matériel détritique grossier (fraction supérieure à $150 \mu \mathrm{m}$ ) et limités à des périodes bien distinctes au cours de la dernière période glaciaire (entre - 60000 et - 10000 ans) ; ces niveaux présentent une certaine périodicité, de l'ordre de 7000 à 10000 ans (figures 7 et 9). La mise en place de ces dépôts, nommés depuis «niveaux de Heinrich » par Broecker et al. (1992), est expliquée par des débâcles massives d'icebergs depuis le détroit d'Hudson et la mer du Labrador, mais aussi depuis la mer de Norvège. En effet, la taille de ces particules détritiques est telle que ni les courants de fond ni les vents ne peuvent expliquer de tels dépôts (Gilbert, 1990). Les icebergs qui arrivent dans l'Atlantique ont entraîné les débris minéraux des moraines prises par les glaces.

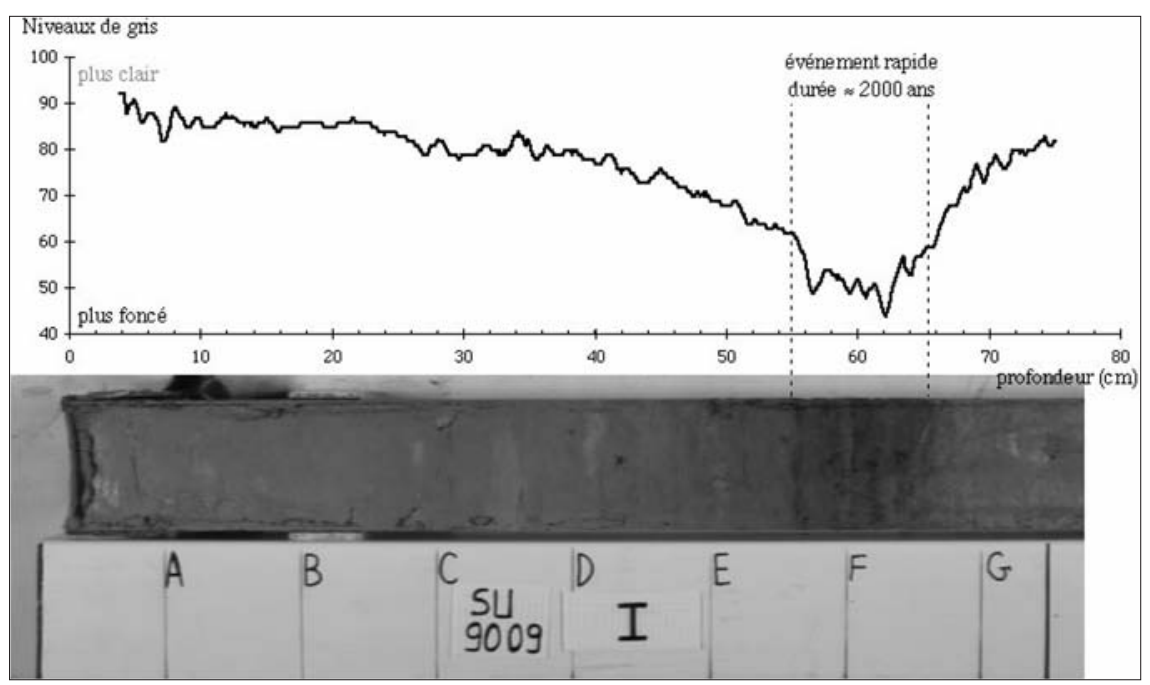

Figure 7 - Photographie d'une demi-carotte de sédiment marin. Les variations de couleur permettent de repérer un niveau plus sombre, dont la durée est estimée à environ 2000 ans. 


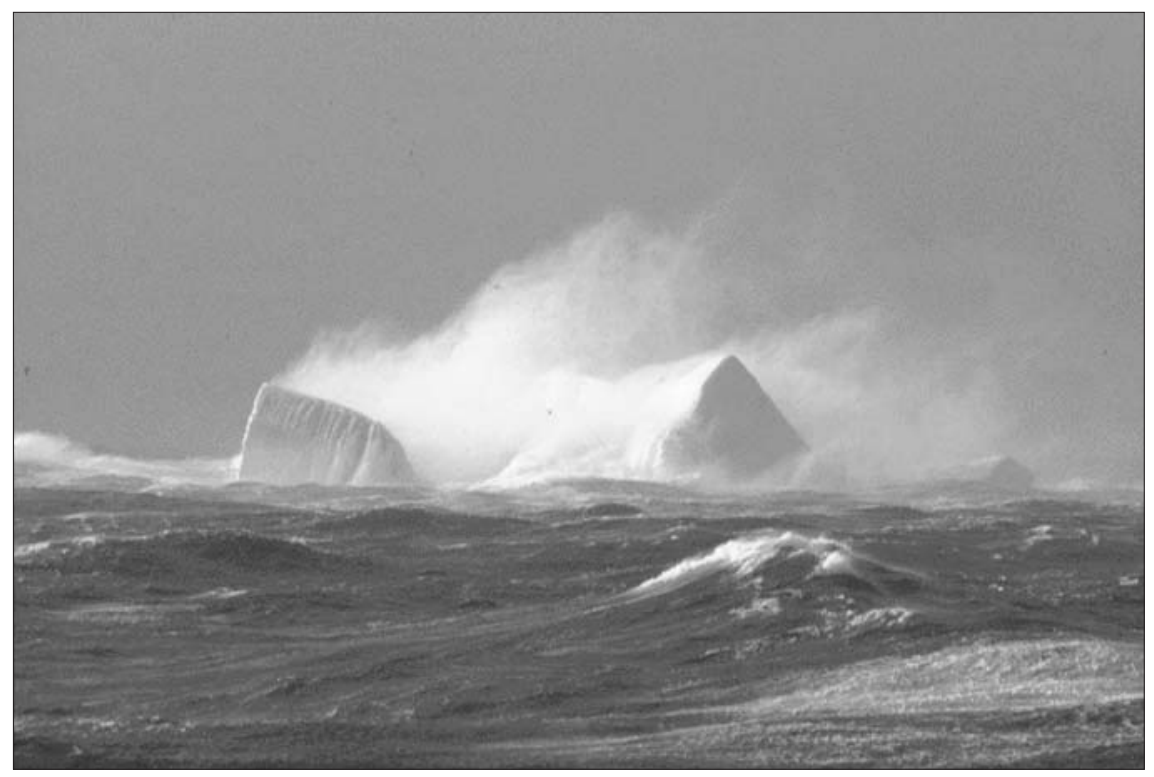

Lorsque les masses de glace fondent en s'approchant des eaux chaudes, les éléments détritiques qu'elles contiennent sont relâchés dans l'océan et sédimentent. Les sédiments marins de la dernière période glaciaire en Atlantique nord sont donc composés d'alternances de niveaux riches en carbonates et en argiles et de niveaux riches en minéraux détritiques grossiers (figure 8).

Les résultats sédimentologiques et magnétiques permettent une bonne caractérisation de ces niveaux, surtout entre 40 et 55 degrés nord. C'est en effet dans cette zone géographique que les valeurs maximales des flux de matériel détritique sont atteintes en période glaciaire (Ruddiman, 1977). Outre le comptage sous loupe binoculaire de la fraction supérieure à $150 \mu \mathrm{m}$ du sédiment, les mesures de la susceptibilité magnétique du sédiment sont un bon moyen d'identifier les niveaux de Heinrich de manière continue (figure 9). Ces niveaux, plus riches en matériau d'origine continentale, ont des propriétés magnétiques très

Figure 8 - Photographie de sédiment marin dans la fraction supérieure à $150 \mu \mathrm{m}$ sous loupe binoculaire. La partie supérieure droite montre un sédiment glaciaire classique, riche en microorganismes carbonatés, les foraminifères, et contenant seulement quelques éléments détritiques. La partie inférieure gauche montre le sédiment d'un niveau de Heinrich, composé essentiellement d'éléments détritiques grossiers.

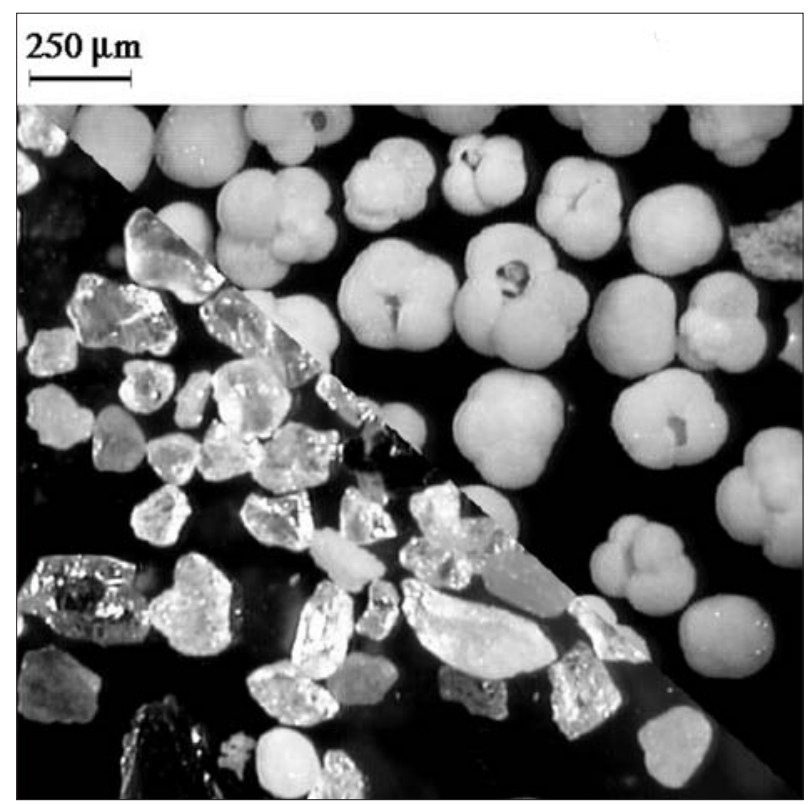

Figure 9 - En haut, enregistrement de la susceptibilité magnétique du sédiment dans la carotte SU90-08, prélevée à $43^{\circ} \mathrm{N}$ et $30^{\circ} \mathrm{W}$. En bas, pourcentage de grains détritiques grossiers (fraction $>150 \mu \mathrm{m}$ ) du sédiment dans la même carotte. Les bandes grises montrent les niveaux de Heinrich.

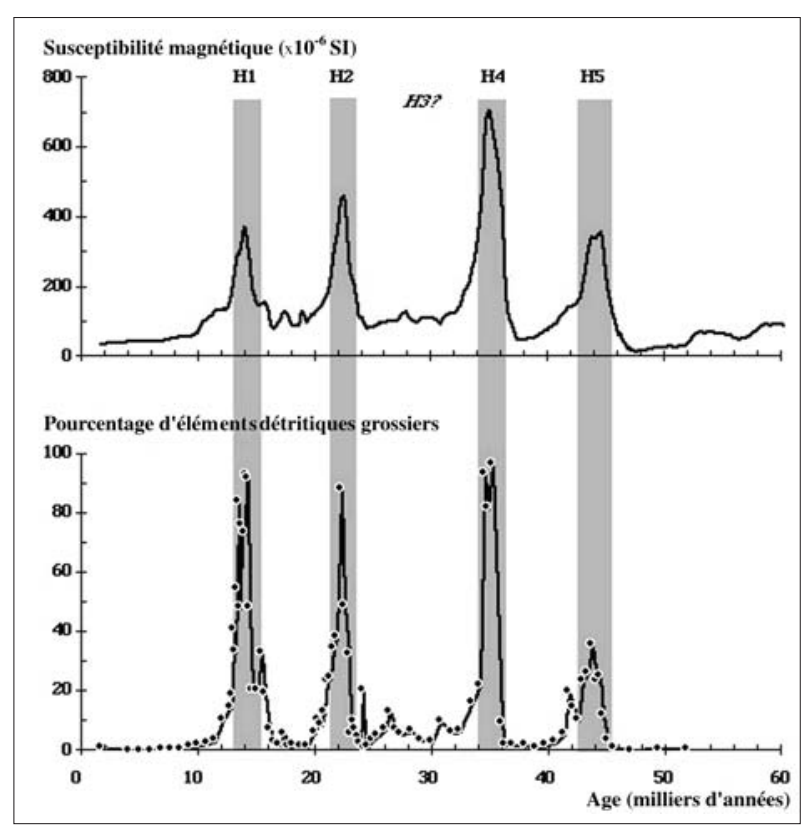


distinctes de celles des sédiments environnants, ce qui permet une identification rapide ; ils sont riches en minéraux magnétiques et ferromagnésiens (magnétite, mais aussi amphiboles, pyroxènes, etc.). On distingue six niveaux de Heinrich, numérotés de H1 à H6 et espacés de 7000 à 10000 ans. Les études de susceptibilité magnétique associées à des études de géochimie isotopique des éléments néodyme et strontium (Grousset et al., 1993) ont permis de proposer des origines et des trajectoires préférentielles pour les icebergs ayant déposé ces niveaux. Les événements H1, H2, H4 et H5 seraient plutôt originaires de l'ancienne calotte Laurentide (recouvrant tout le nord de l'Amérique du Nord), alors que les événements H3 et H6 auraient plutôt une origine fenno-scandinave via la mer de Norvège.

Lorsque l'on étudie la composition isotopique de l'espèce polaire de foraminifère planctonique Neogloboquadrina pachyderma à enroulement senestre dans les mêmes niveaux, on constate que chacun des pics de susceptibilité magnétique est associé à un minimum de composition isotopique (figure 10). Des minima isotopiques peuvent en théorie correspondre, soit à une augmentation de température, soit à une arrivée d'eau de fonte. Nous avons démontré préalablement par d'autres paramètres que nous étions en présence de décharges d'icebergs. Chacun de ces minima isotopiques traduit donc une décharge d'icebergs de grande ampleur qui a affecté la totalité de l'Atlantique nord.

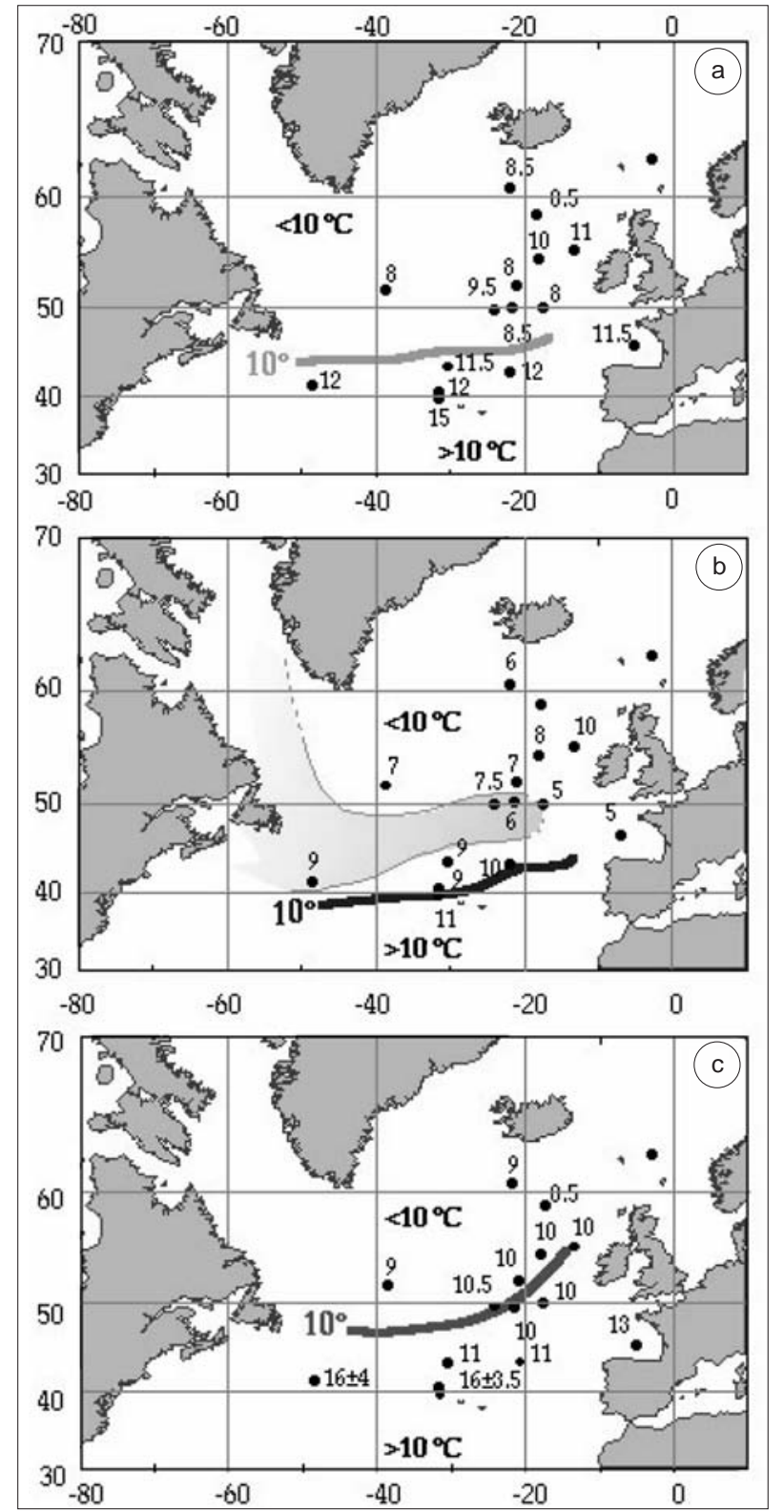

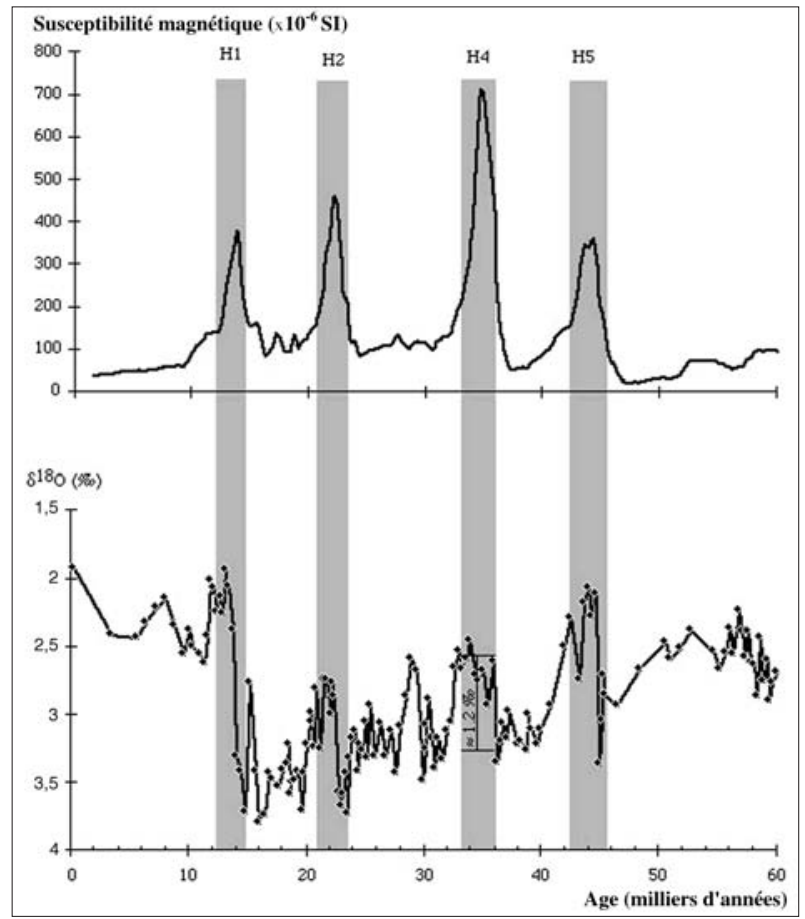

$\Delta$

Figure 10 - Pour la carotte SU90-08, comparaison entre l'enregistrement de la susceptibilité magnétique du sédiment et celui de la composition isotopique de l'oxygène (exprimée sous forme de teneur relative en ${ }^{18} \mathrm{O}$ des foraminifères planctoniques $N$. pachyderma senestre). Mise en évidence des niveaux de Heinrich. Pour le niveau de Heinrich 4, I'amplitude du pic isotopique est indiquée.

Figure 11 - Cartes de reconstitution de la température des eaux de surface en été pour trois dates:

- en haut(a), avant la débâcle à - 37000 ans ;

- au centre (b), pendant la débâcle à - 35000 ans ; est également reportée la zone de fonte maximale des icebergs telle que reconstruite à partir de la composition isotopique de l'oxygène ;

- en bas (C), après la débâcle à - 33000 ans.

$\mathrm{L}$ 'isotherme $10{ }^{\circ} \mathrm{C}$, qui délimite les eaux polaires des eaux subpolaires en été, a été tracée sur chaque carte. 


\section{Les reconstitutions de la température des eaux de surface du passé}

Les reconstitutions de la température des eaux de surface sont obtenues en utilisant le principe d'actualisme : les écosystèmes passés sont comparables aux écosystèmes modernes et les espèces anciennes de foraminifères - dans la limite des deux derniers cycles climatiques, soit environ 300000 années - ont la même biologie que les modernes. Pratiquement, on estime que la composition en foraminifères des sommets de carottes modernes est liée à la nature physique des masses d'eau sus-jacentes. Dans un océan donné, nous connaissons les différentes espèces de foraminifères présentes dans les sédiments modernes et la température des eaux de surface correspondantes. La méthode consiste donc à chercher dans un ensemble de sédiments modernes les lieux pour lesquels la composition en faune sera la plus proche de celle des niveaux anciens étudiés (Prell, 1985). La température ancienne est alors obtenue par comparaison avec les données modernes de température. La barre d'erreur sur de telles reconstructions est d'environ \pm 1 à $1,5^{\circ} \mathrm{C}$.

La composition isotopique des foraminifères planctoniques dépend des variations planétaires du volume des glaces, de la température de l'eau de formation et de la composition isotopique de l'eau de mer, donc de la salinité. La température est déterminée par les différentes espèces de foraminifères planctoniques présentes et les variations du volume des glaces sont reconstituées à partir de séries de référence. Il est alors possible de reconstituer l'impact sur la salinité des eaux de surface de telles décharges (figure 3).

Pour mener à bien ce travail, nous avons choisi d'étudier plus particulièrement l'événement de Heinrich 4, daté à environ - 35000 ans par le carbone 14. Cet événement offre l'avantage d'être bien enregistré et visible sur toutes les carottes étudiées, et d'être bien déterminé stratigraphiquement grâce au carbone 14. De plus, entre - 30000 et - 40000 ans, les paramètres de l'insolation ne montrent aucune variation importante, ce qui traduit une certaine stabilité de la quantité d'énergie reçue par la Terre et permet d'avoir une vision simplifiée de ce qu'est un tel événement. D'autre part, les variations du volume de glace à l'échelle du globe (liées aux alternances glaciaire-interglaciaire) sont négligeables pendant cette période.

Dans un premier temps, il a fallu estimer les variations de la température de l'eau en surface de l'océan provoquées par la décharge d'icebergs associée à cet événement, en utilisant la méthode décrite dans l'encadré ci-dessus. Pour cela, nous avons choisi de considérer trois dates situées à - 37000 , - 35000 et - 33000 ans, soit respectivement avant, pendant et après la décharge (figure 11). La reconstitution montre que les valeurs de température de surface de l'eau, en été, ont diminué de 2 à $6{ }^{\circ} \mathrm{C}$ (valeurs extrêmes atteintes en bordure du continent européen) pendant l'événement proprement dit. À - 33000 ans, la situation est à nouveau similaire à ce qu'elle était avant la débâcle, mais les températures sont toutes, en moyenne, de 1 à $1,5^{\circ} \mathrm{C}$ plus chaudes (Cortijo et al., 1997).

Simultanément à cette étude de température, on a aussi estimé l'impact du flux d'eau douce sur la salinité des eaux de surface. Une approximation de la réponse de la salinité des eaux de surface peut être obtenue à partir de l'amplitude du pic isotopique de l'événement de Heinrich 4 (figure 10) : en effet, dans ce cas, le signal isotopique est essentiellement dû à l'arrivée massive d'eau de fonte ; la différence entre le niveau du maximum et celui du minimum nous donne une estimation de ce qu'a pu être la variation de salinité associée. C'est ce qui est reporté sur la figure 11b. L'amplitude du pic isotopique associé à la décharge d'icebergs est maximale entre 45 et 50 degrés nord, zone où les icebergs entrent en contact avec les eaux chaudes du Gulf Stream. Les estimations de salinité associées à cette décharge d'icebergs indiquent une diminution de la salinité des eaux de surface de l'ordre de 1 à $3 \%$.

Cette étude géographique réalisée sur l'événement de Heinrich 4 a été étendue à l'ensemble des événements de Heinrich enregistrés sur 60000 ans dans une même carotte (à 43 degrés nord et 30 degrés ouest). Les conclusions obtenues sont comparables : diminution en moyenne de 2 à $3{ }^{\circ} \mathrm{C}$ de la température et de 1 à $3 \%$ de la salinité des eaux de surface à chacun des événements. Ces diminutions sont loin d'être sans conséquences sur la circulation océanique profonde, 
Représentation schématique de la circulation thermohaline. Flèches gris foncé : courant chaud de surface. Flèches gris clair : courant profond froid et salé.

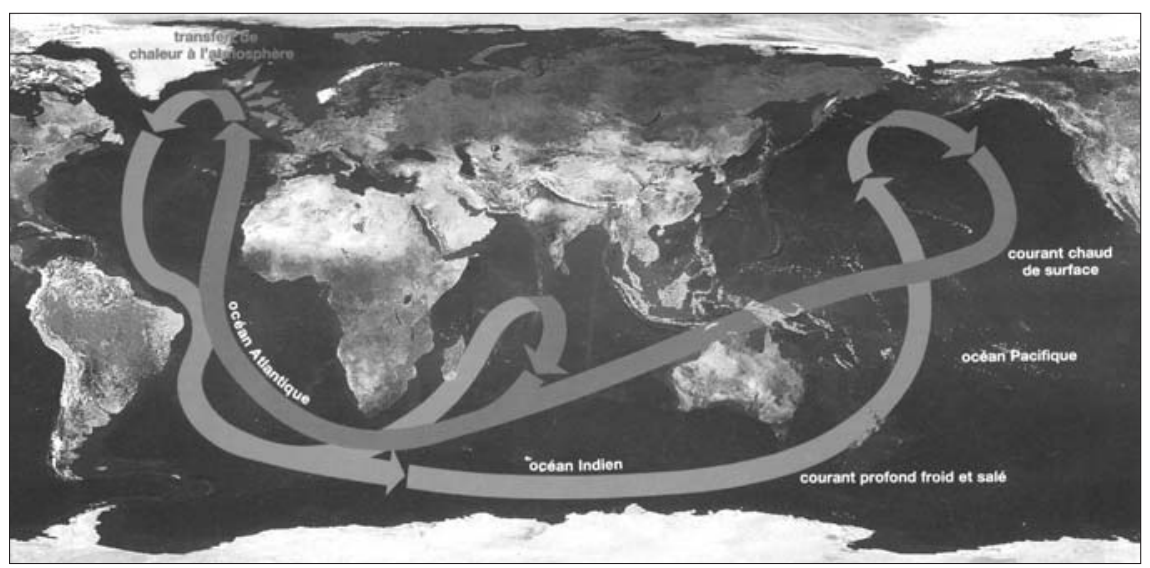

pour laquelle le nord de l'Atlantique est une zone moteur. En effet, c'est en mer de Norvège que les eaux de surface relativement salées en provenance des zones chaudes de l'Atlantique plongent par augmentation de densité et alimentent les bassins océaniques profonds de tout le globe. Cette circulation océanique a connu des changements notables au cours de la dernière période glaciaire et Vidal et al. (1997) ont montré qu'à chaque décharge d'icebergs correspondait un ralentissement de la circulation océanique dû à la diminution de densité des eaux de surface.

En résumé, les événements de Heinrich, au nombre de six entre - 60000 et - 10000 ans, sont engendrés par des débâcles pseudo-périodiques des calottes de Laurentide et de Fennoscandie (débâcles de la Laurentide principalement pour les événements 1, 2, 4 et 5 ; débâcles de la Fennoscandie pour les événements 3 et 6). La conséquence de ces débâcles est une diminution de la température de l'ordre de 2 à $3{ }^{\circ} \mathrm{C}$ et de la salinité de 1 à $3 \%$ pour les eaux de surface entre 40 et 60 degrés nord. Ces changements hydrologiques ont pour résultat de faire décroître la densité des eaux de surface, et donc de ralentir la circulation océanique dans l'Atlantique nord. Cela entraîne des modifications climatiques dans tout le bassin de l'Atlantique nord et sur les continents environnants, avec des variations de température de l'air de plusieurs degrés, par réduction de l'apport de chaleur aux hautes latitudes. De nouvelles études montrent que le climat pourrait même être affecté à l'échelle de la planète, puisque des événements comparables sont retrouvés dans le Pacifique ou en mer d'Arabie (Behl et Kennett, 1996 ; Sirocko et al., 1996).

Lorsque l'on considère, pendant la même période de temps, les variations de la

LES ÉVÉNEMENTS DE DANSGAARD-OESCHGER température de l'air enregistrées dans la glace de la calotte polaire du Groenland, on constate que la variabilité ne se limite pas à six événements au cours des 60000 dernières années. Les glaciologues ont en effet identifié, pour cette même période, dix-huit événements présentant les mêmes caractéristiques (Dansgaard et al., 1993) ; ces événements ont été baptisés interstades ou événements de Dansgaard-Oeschger (figure 12). Comme je l'ai mentionné précédemment, le nord de l'Atlantique nord et la mer de Norvège sont des zones clés pour la circulation océanique : tout changement de la température de l'air au-dessus du Groenland est lié à un changement de la circulation océanique. Nous allons donc étudier une carotte sédimentaire en mer de Norvège pour suivre l'évolution hydrologique à proximité du Groenland.

Comme nous l'avons vu dans la partie précédente, la susceptibilité magnétique des carottes sédimentaires prélevées entre 40 et 55 degrés nord montre des valeurs maximales à chacun des événements de Heinrich. En mer de Norvège, les enregistrements de ce même paramètre sont très différents (figure 13) :

- Aux moyennes latitudes de l'hémisphère nord, les événements de Heinrich sont identifiables dans les courbes de susceptibilité magnétique par des pics qui signalent l'arrivée de matériel d'origine continentale dans un sédiment carbonaté peu ou pas magnétique. 


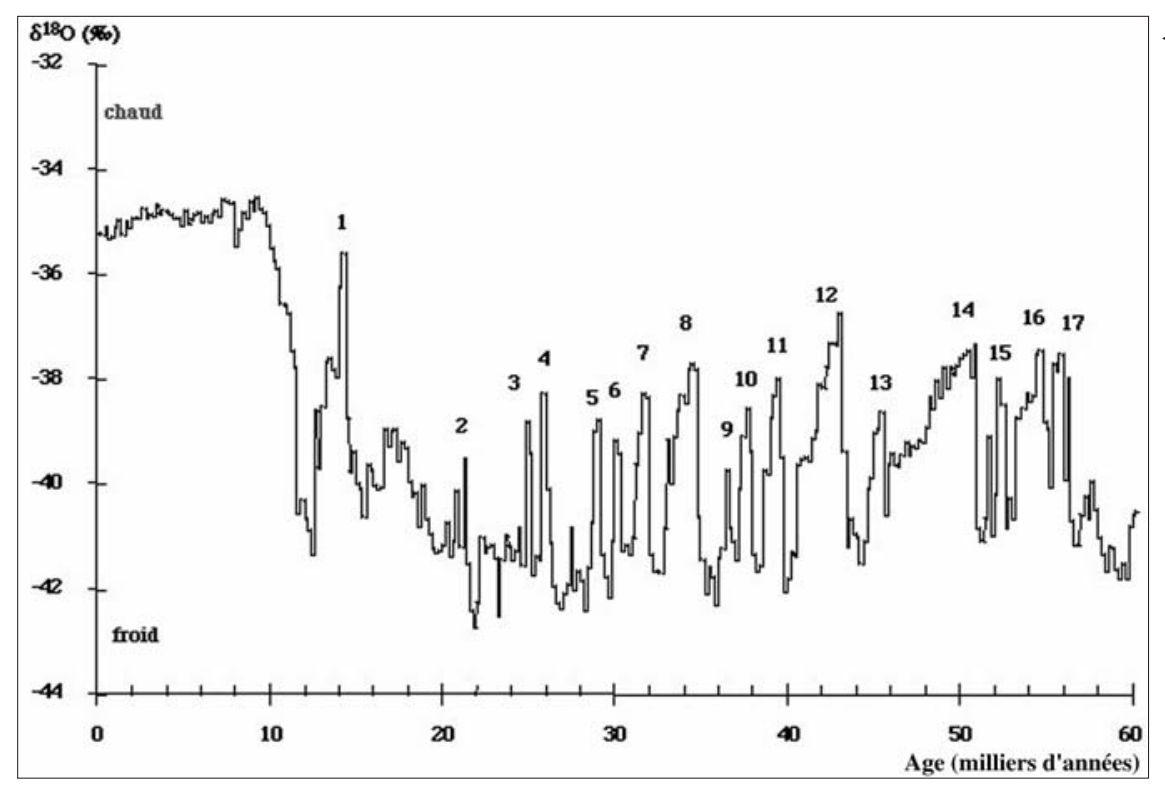

Figure 12 - Enregistrement de la composition isotopique de l'oxygène (exprimée sous forme de teneur relative en ${ }^{18} \mathrm{O}$ ) dans la glace au site Grip au Groenland (Dansgaard et al., 1993). Les chiffres indiquent les divers interstades.

Figure 14 - Enregistrements de la composition isotopique de l'oxygène du foraminifère planctonique polaire $N$. pachyderma à enroulement senestre (en bas) et de la susceptibilité magnétique du sédiment (au centre) dans la carotte ENAM93-21, comparés à l'enregistrement isotopique de la carotte glaciaire de Grip (en haut). Les niveaux de Heinrich sont repérés et les interstades glaciaires reportés sur l'enregistrement de susceptibilité magnétique.
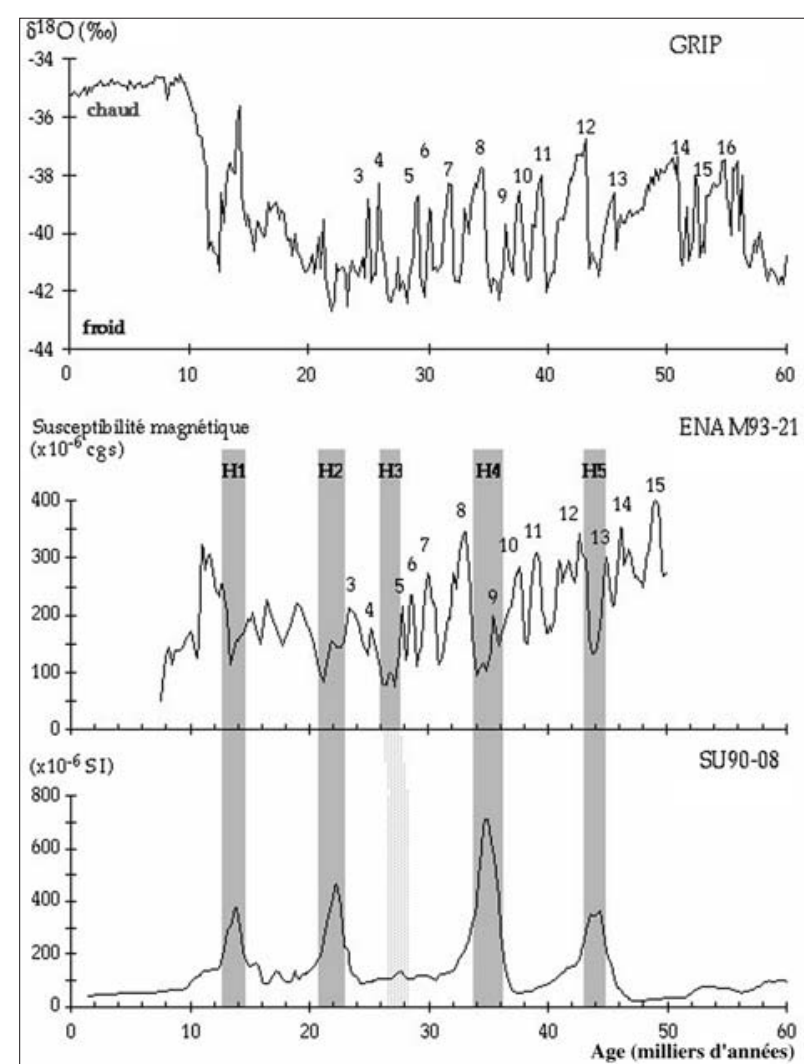

Figure 13 - Comparaison entre l'enregistrement isotopique de la carotte glaciaire prélevée à Grip (en haut) et les enregistrements de susceptibilité magnétique des carottes sédimentaires prélevées à $62^{\circ} \mathrm{N}$ (carotte ENAM93-21, d'après Rasmussen et al. [1996a], au centre), et à $43^{\circ} \mathrm{N}$ (carotte SU90-08, en bas). Les chiffres correspondent aux interstades identifiés dans Grip.

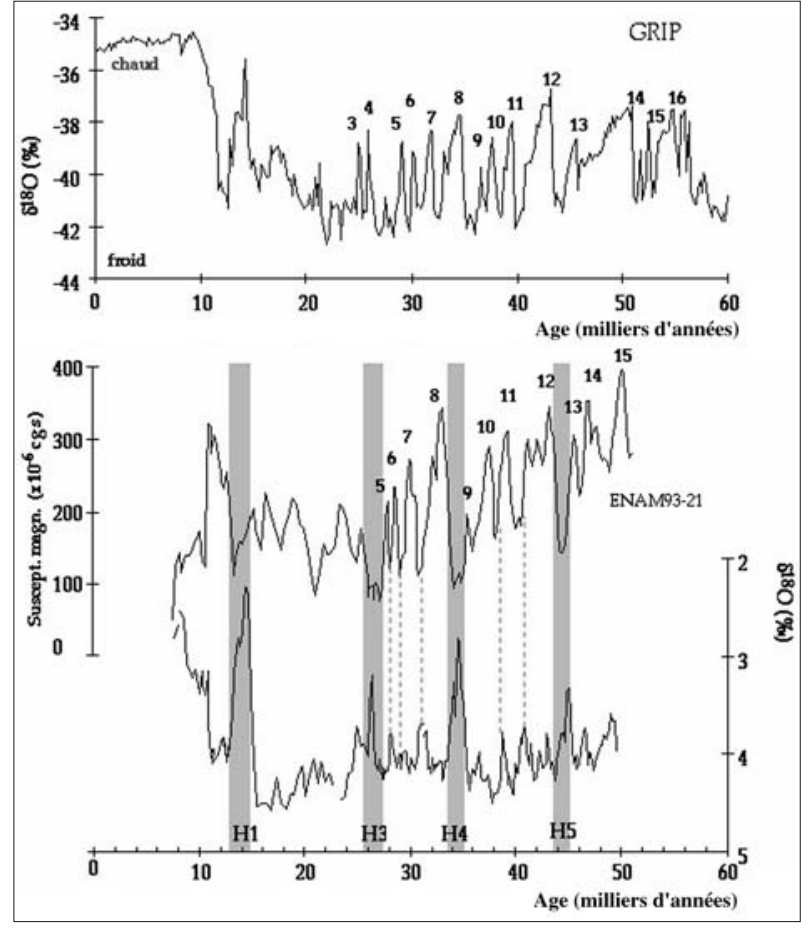

- En mer de Norvège, la composition des sédiments est très différente et les événements de Heinrich sont identifiables par des creux dans les courbes, soit un signal opposé à celui enregistré plus au sud. Cela vient en partie du fait que le sédiment y est toujours plus riche en matériel détritique.

Le caractère remarquable de cet enregistrement de la mer de Norvège tient à sa très grande ressemblance avec l'enregistrement de la composition isotopique de la glace au Groenland : les interstades 3 à 18 repérés par les glaciologues dans la glace peuvent également être identifiés dans l'enregistrement de susceptibilité magnétique de la carotte de sédiment marin (figure 13).

Bien que les signatures de la susceptibilité magnétique dans les carottes soient très différentes entre moyennes et hautes latitudes, la comparaison des enregistrements isotopiques montre que les changements hydrologiques associés aux 
débâcles d'icebergs présentent la même signature quel que soit le contexte hydrologique et climatique d'ensemble (figure 14). Chacun des creux dans l'enregistrement de susceptibilité magnétique correspond à une diminution de la composition isotopique de l'oxygène, ce qui traduit une variation concomitante de l'origine des éléments magnétiques et de l'hydrologie de surface. En mer de Norvège, nous observons ainsi à la fois les enregistrements des événements de Heinrich et ceux des événements de Dansgaard-Oeschger initialement mis en évidence dans la glace.

L'ensemble de ces observations permet de dresser des hypothèses de mise en place pour les différents types d'événements.

HYPOTHÈSES DE MISE EN PLACE
Les mécanismes associés aux débâcles d'icebergs en Atlantique nord et en mer de Norvège et les liens entre ces deux types de phénomènes restent encore à élucider. La calotte de glace est un élément essentiel du système climatique pendant la période glaciaire et il est important de connaître les différents processus qui peuvent l'affecter au cours d'un événement rapide. À l'issue de ce travail, un modèle a été proposé pour expliquer le comportement des calottes au cours d'un événement rapide (figure 15) ; ce modèle s'appuie sur des travaux effectués par Denton et al. (1986) et MacAyeal (1993).

Quand le volume de glace est minimal, les eaux de surface de l'Atlantique nord et du sud de la mer de Norvège sont relativement chaudes et les précipitations permettent l'augmentation progressive du volume de glace de la Laurentide et de la Fennoscandie. L'enregistrement isotopique de la température de l'atmosphère située au-dessus du Groenland montre un refroidissement progressif et les

\section{(a)}

\section{Dynamique liée à la calotte de glace de la Laurentide}

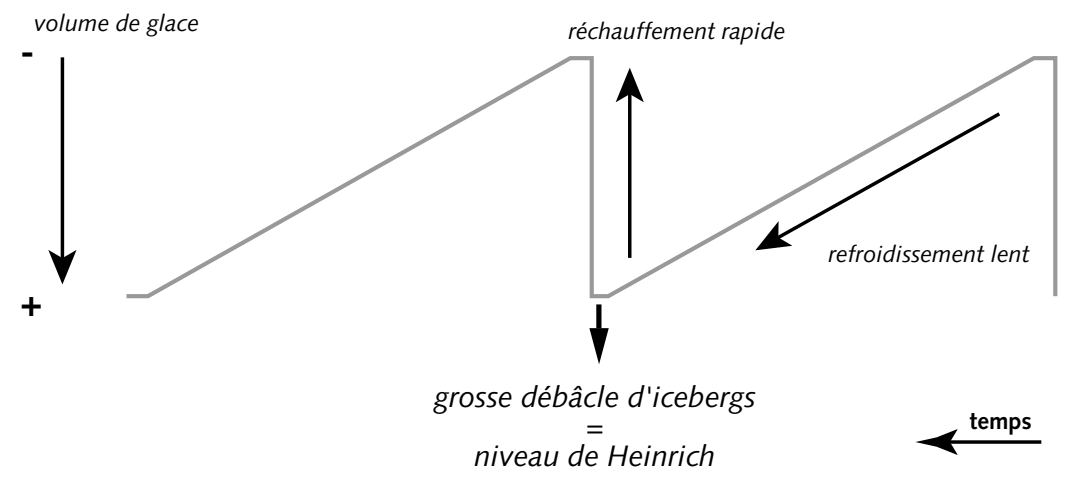

(b)

\section{Dynamique liée aux calottes de glace de Fennoscandie et du Groenland}

oscillations rapides de la température de l'air événements de Dansgaard-Oeschger

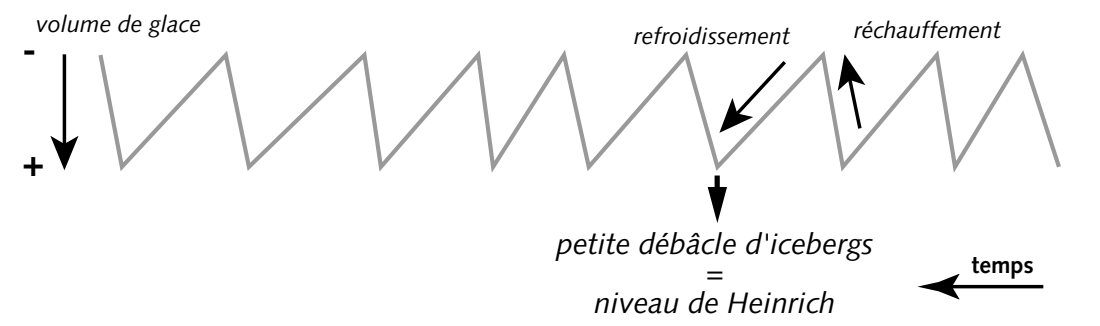

Figure 15 - Schémas hypothétiques de la variation du volume des calottes glaciaires en fonction du temps et de son impact sur les débâcles d'icebergs et sur la température de l'air. (a) pour la mise en place des niveaux de Heinrich :

(b) pour la mise en place des niveaux de Dansgaard-Oeschger. 
masses d'eau polaires se déplacent vers le sud. Lorsque le volume des glaces atteint un certain seuil, la calotte de Laurentide se déstabilise et se désagrège en icebergs qui se répandent sur tout le nord de l'Atlantique et y relâchent des éléments détritiques. Un niveau de Heinrich est alors enregistré dans les sédiments marins. La circulation thermohaline est affectée et ralentie par cette arrivée de glace et donc d'eau de fonte, à cause de la diminution de densité liée aux variations de température et de salinité de l'eau. Lorsque la majeure partie de cette eau douce est résorbée, la circulation thermohaline se rétablit et un réchauffement se produit dans les systèmes océanique et atmosphérique (Paillard et Labeyrie, 1994).

Les instabilités de la Fennoscandie (et peut-être du Groenland) sont plus fréquentes (tous les 2000 ans environ) que celles de la Laurentide, mais de moins grande amplitude, probablement à cause du plus petit volume des calottes glaciaires ou de l'absence de grands couloirs d'érosion comme la baie de Hudson pour la Laurentide. L'enregistrement des débâcles de la Fennoscandie dans les sédiments, au nord de 50 degrés nord, correspond aux événements de DansgaardOeschger. Ces oscillations se superposent aux larges oscillations climatiques provoquées par la Laurentide dans une bande de latitude comprise entre 50 et 62 degrés nord environ.

\section{BILAN ET PERSPECTIVES}

Une des questions majeures subsistant à l'issue de cette étude concerne le moteur de cette variabilité rapide. Les premiers auteurs (Heinrich, 1988) avaient associé les débâcles d'icebergs des niveaux de Heinrich à une manifestation du demi-cycle astronomique de la précession. Cependant, les calottes glaciaires sont un élément essentiel du système climatique à cette période et les différents mécanismes qui peuvent l'affecter sont sans aucun doute importants pour comprendre cette variabilité.

Les événements rapides ont été étudiés pour la dernière période glaciaire, pendant laquelle les conditions d'insolation sont à la fois connues et différentes de ce qu'elles ont été au cours des précédentes périodes glaciaires. Il serait aussi très intéressant d'étudier une période pendant laquelle l'insolation était très différente de ce qu'elle était au cours de ces 60000 dernières années, de façon à pouvoir estimer l'influence de ce paramètre dans la variabilité climatique rapide. En effet, si la pseudo-périodicité à environ 10000 ans observée dans les événements de Heinrich est liée au cycle de la précession, elle devrait disparaître dans une période où la précession était moins importante pour les modulations climatiques planétaires. Par contre, si cette périodicité était conservée, cela pourrait vouloir dire que le moteur de la variabilité rapide est bien la dynamique interne des calottes de glace.

De nombreuses études, tant sur le plan expérimental qu'en modélisation numérique, seront encore nécessaires pour comprendre les mécanismes et l'impact planétaire de telles décharges d'icebergs. Plusieurs campagnes océanographiques sont d'ores et déjà en cours de préparation pour collecter du matériel dans des zones de l'océan déterminantes pour la compréhension des mécanismes qui régulent et déséquilibrent le système climatique. Mais, si les variations climatiques rapides sont explicables en période glaciaire par des débâcles d'icebergs provenant des grandes calottes de glace sur les continents (il nous reste toutefois beaucoup d'inconnues), de telles variations climatiques sont encore bien mal connues en période interglaciaire.

Selon la théorie astronomique des climats, la période interglaciaire que nous connaissons actuellement devrait lentement évoluer vers une période glaciaire. Mais les résultats des mesures instrumentales tendent à montrer que la température de l'air augmente. Ce réchauffement, attribué à l'augmentation des gaz à effet de serre due aux activités humaines, devrait se poursuivre durant le prochain siècle puisque les différents indicateurs mesurés dans les glaces indiquent que, dans le passé, la température de l'air a évolué parallèlement à la concentration en gaz à effet de serre. Mais la méconnaissance de la variabilité naturelle du climat en période interglaciaire rend difficile toute prévision de l'évolution future. En effet, une simple fluctuation du bilan de l'évaporation et des précipitations au-dessus de l'Atlantique nord pourrait causer un ralentissement ou une amplification du réchauffement planétaire actuellement envisagé pour le prochain siècle, jouant ainsi un rôle climatique analogue à celui des débâcles d'icebergs dans la même zone géographique durant les périodes glaciaires. 
Remerciements

BIBLIOGRAPHIE
Je tiens à remercier les différentes personnes qui m'ont guidée dans ce travail de thèse au Centre des faibles radioactivités, et notamment MM. Laurent Labeyrie et Jean-Claude Duplessy. Je remercie également M. Didier Renaut de son aide pour la relecture de ce manuscrit. Cet article porte le numéro de contribution LSCE 2018.

Bard E., M. Arnold, J. Mangerud, M. Paterne, L. Labeyrie, J. Duprat, M.-A. Melières, E. Sonstegaard et J.-C. Duplessy, 1994 : The North Atlantic atmosphere-sea surface ${ }^{14} \mathrm{C}$ gradient during the Younger Dryas climatic event. Earth and Planetary Science Letters, 126, 275-287.

Behl R. J. et J.-P. Kennett, 1996 : Brief interstadial events in the Santa Barbara basin, NE Pacific, during the past 60 kyr. Nature, 379, 243-246.

Bond G., H. Heinrich, W. S. Broecker, L. Labeyrie, J. McManus, J. Andrews, S. Huon, R. Jantschik, S. Clasen, C. Simet, K. Tedesco, M. Klas, G. Bonani et S. Ivy, 1992 : Evidence for massive discharges of icebergs into the North Atlantic Ocean during the last glacial period. Nature, 360, 245-251.

Bond G., W. Broecker, S. Johnsen, J. McManus, L. Labeyrie, J. Jouzel et G. Bonani, 1993 : Correlations between climate records from North Atlantic sediments and Greenland ice. Nature, 365, 143-147.

Broecker W., G. Bond, M. Klas, E. Clark et J. McManus, 1992 : Origin of the northern Atlantic's Heinrich events. Climate Dynamics, 6, 265-273.

Cortijo E., L. Labeyrie, L. Vidal, M. Vautravers, M. Chapman, J.-C. Duplessy, M. Elliot, M. Arnold, J.-L. Turon et G. Auffret, 1997 : Changes in sea surface hydrology associated with Heinrich event 4 in the North Atlantic Ocean between $40^{\circ} \mathrm{N}$ and $60^{\circ} \mathrm{N}$. Earth and Planetary Science Letters, 146, 29-45.

Dansgaard W., H. B. Clausen, N. Gundestrup, C. U. Hammer, S. F. Johnsen, P. M. Kristinsdottir et N. Reeh, 1982 : A new Greenland deep ice core. Science, 218, 1273-1277.

Dansgaard W., S. J. Johnsen, H. B. Clausen, D. Dahl-Jensen, N. S. Gundestrup, C. U. Hammer, C. S. Hvidberg, J. P. Steffensen, A. E. Sveinbjörnsdottir, J. Jouzel et G. Bond, 1993 : Evidence for general instability of past climate from a 250-kyr ice-core record. Nature, 364, 218-220.

Denton G. H., T. J. Hughes et W. Karlen, 1986 : Global ice-sheet system interlocked by sea level. Quaternary Research, 26, 3-26.

Emiliani C., 1955 : Pleistocene temperatures. Journal of Geology, 63, 538-578.

Epstein S., R. Buchsbaum, H. A. Lowenstam et H. C. Urey, 1953 : Revised carbonatewater isotopic temperature scale. Geological Society of America Bulletin, 64, 1315-1325.

Fillon R. H. et J.-C. Duplessy, 1980 : Labrador sea bio-, tephro-, oxyden isotopic stratigraphy and Late Quaternary paleoceanographic trends. Canadian Journal of Earth Sciences, 17, 831-854.

Fronval T., E. Jansen, J. Bloemendal et S. Johnsen, 1995 : Oceanic evidence for coherent fluctuations in Fennoscandian and Laurentide ice sheets on millenium timescales. Nature, $374,443-446$.

Gilbert R., 1990 : Rafting in glacimarine environments, in Dowdeswell J. A. et J. D. Scourse ed., Glacimarine environments: Processes and sediments, volume 53, Geological Society, 105-120.

Grousset F. E., L. Labeyrie, J. A. Sinko, M. Cremer, G. Bond, J. Duprat, E. Cortijo et S.

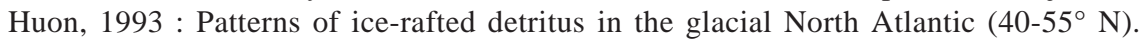
Paleoceanography, 8, 175-211.

Hays J. D., J. Imbrie et N. J. Shackleton, 1976 : Variations in the Earth's Orbit: Pacemaker of the Ice Ages. Science, 194, 1121-1132.

Heinrich H., 1988 : Origin and consequences of cyclic ice-rafting in the Northeast Atlantic Ocean during the past 130,000 years. Quaternary Research, 29, 142-152.

Hillaire-Marcel C., A. de Vernal, G. Bilodeau et G. Wu, 1994 : Isotope stratigraphy, sedimentation rates, deep circulation, and carbonate events in the Labrador Sea during the last 200 ka. Canadian Journal of Earth Science, 31, 63-89. 
MacAyeal D. R., 1993 : A low-order model of the Heinrich event cycle. Paleoceanography, 8, 767-773.

Martinson D. G., N. G. Pisias, J. D. Hays, J. Imbrie, T. C. Moore et N. J. Shackleton, 1987 : Age dating and the orbital theory of the ice ages: development of a high-resolution 0 to 300,000 year chronostratigraphy. Quaternary Research, 27, 1-29.

Maslin M. A., N. J. Shackleton et U. Pflaumann, 1995 : Surface water temperature, salinity and density changes in the northeast Atlantic during the last 45,000 years: Heinrich events, deep water formation and climatic rebounds. Paleoceanography, 10, 527-544.

Mix A. C. et R. G. Fairbanks, 1985 : North Atlantic surface-ocean control of Pleistocene deep-ocean circulation. Earth and Planetary Science Letters, 73, 231-243.

Paillard D. et L. Labeyrie, 1994 : Role of the thermohaline circulation in the abrupt warming after Heinrich events. Nature, 372, 162-164.

Pastouret L., G. A. Auffret, M. Hoffert, M. Melguen, H. D. Needham et C. Latouche, 1975 : Sédimentation sur la ride de Terre-Neuve. Canadian Journal of Earth Sciences, 12, 1019-1035.

Prell W. L., 1985 : The stability of low-latitude sea-surface temperatures: an evolution of the Climap reconstruction with emphasis on the positive SST anomalies. United States Department of Energy.

Rasmussen T. L., E. Thomsen, T. C. E. Van Weering et L. Labeyrie, 1996a : Rapid changes in surface and deep water conditions at the Faeroe Margin during the last 58,000 years. Paleoceanography, 11, 757-771.

Rasmussen T. L., T. C. E. Van Weering et L. Labeyrie, 1996b : High resolution stratigraphy of the Faeroe-Shetland Channel and its relation to North Atlantic paleoceanography: the last 87 kyr. Marine Geology, 131, 75-88.

Ruddiman W. F., 1977 : Late Quaternary deposition of ice-rafted sand in the subpolar North Atlantic. Geological Society of America Bulletin, 88, 1813-1827.

Ruddiman W. F. et L. K. Glover, 1972 : Vertical mixing of ice-rafted volcanic ash in North Atlantic sediments. Geological Society of America Bulletin, 83, 2817-2836.

Ruddiman W. F. et A. McIntyre, 1981 : The North Atlantic Ocean during the last deglaciation. Palaeogeography, Palaeoclimatology, Palaeoecology, 35, 145-214.

Shackleton N. J., 1974 : Attainment of isotopic equilibrium between ocean water and benthonic foraminifera genus Uvigerina: isotopic changes in the ocean during the last glacial. Dans Les méthodes quantitatives d'étude des variations du climat au cours du Pleistocène. CNRS, Gif-sur-Yvette, 203-209.

Sirocko F., D. Garbe-Schönberg, A. McIntyre et B. Molfino, 1996 : Teleconnections between the subtropical monsoons and high-latitude climates during the last deglaciation. Science, 272, 526-529.

Van Kreveld S.A., M. Knappertsbusch, J. Ottens, G. M. Ganssen et J. E. Van Hinte, 1996 : Biogenic carbonate and ice-rafted debris (Heinrich layer) accumulation in deep-sea sediments from a Northeast Atlantic piston core. Marine Geology, 131, 21-46.

Vidal L., L. Labeyrie, E. Cortijo, M. Arnold, J.-C. Duplessy, E. Michel, S. Becqué et T. C. E. Van Weering, 1997 : Evidence for changes in the North Atlantic Deep Water linked to meltwater surges during the Heinrich events. Earth and Planetary Science Letters, 146, 13-26.

\section{Glossaire}

Bioturbation : perturbation de l'organisation initiale d'un sédiment par les déplacements de micro-organismes vivants enfouis ou posés sur le fond des océans. La disposition initiale des couches sédimentaires va être modifiée et les informations qu'elles contiennent peuvent être dégradées.

Circulation thermohaline : la circulation océanique est principalement induite par des gradients de densité dus à l'effet combiné des variations de la température et de la salinité. Les eaux du Gulf Stream, puis de la dérive nord-atlantique, chaudes et salées, sont transportées aux hautes latitudes de I'hémisphère nord et libèrent leur chaleur dans l'atmosphère. En mer de Norvège, l'augmentation de densité 
qui va provoquer la plongée des eaux de surface est liée à l'effet combiné, en hiver, du refroidissement des eaux de surface et de la formation de glace de mer (la glace de mer se forme à partir d'eau douce et l'eau restante est alors enrichie en sel). Les eaux profondes ainsi formées vont envahir les bassins océaniques profonds et parcourir la totalité des fonds océaniques jusqu'au Pacifique. La circulation océanique planétaire prend donc sa source en mer de Norvège dans l'hémisphère nord et en mer de Weddel dans l'hémisphère sud. La mer de Norvège est ainsi une zone clé pour la circulation océanique planétaire : si cette plongée d'eau profonde est ralentie, c'est tout le système mondial de circulation océanique qui est affecté.

Foraminifères : les foraminifères sont des micro-organismes unicellulaires essentiellement marins, dont la coquille peut être carbonatée ou chitineuse. Ils peuvent mesurer de quelques centaines de micromètres à quelques millimètres. Pour les études paléoclimatiques, seuls les foraminifères à coquille carbonatée sont utilisés. On différencie les foraminifères planctoniques vivant à la surface de l'océan (généralement dans les cent premiers mètres) des foraminifères benthiques qui vivent posés sur le fond ou enfouis dans les premiers millimètres des sédiments marins.

Interstades : dénomination adoptée par les glaciologues pour désigner les épisodes chauds observés dans les enregistrements de la composition isotopique de la glace du Groenland entre - 110000 et - 10000 ans avant nos jours ; ces épisodes sont numérotés de 1 à 21 .

Matériel détritique grossier : le matériel détritique grossier (fraction supérieure à $150 \mu \mathrm{m})$ des sédiments marins est constitué de minéraux érodés et transportés dans l'océan depuis les continents adjacents. Ces minéraux sont essentiellement des quartz, des feldspaths, des micas, des minéraux ferromagnésiens et magnétiques.

Principe d'actualisme : ce principe est à la base de nombreuses études paléoclimatiques. Les écosystèmes actuels répondent à des spécificités physiques et chimiques de l'environnement (température, salinité, etc.). On considère que, dans les limites données par l'évolution des espèces, les conditions n'ont pas changé pour les mêmes espèces au cours des temps.

Stratigraphie : discipline qui, à partir de différents indices, établit des corrélations entre diverses séries sédimentaires pour les placer sur une même échelle de temps. 